\title{
PICA LEADING TO LITHOBEZOAR AND TRICHOBEZOAR IN ADULT MALE: A CASE REPORT
}

KEY WORDS: Pica, Bezoars, Small Bowel Obstruction, Intellectual Disability

\section{Dr Bhuvana}

Lakshmi

Sundararajan

Dr Siddartha Gowthaman*

Dr Arul Kumar

\section{Dr Ramanathan}

M

분

INTRODUCTION: Pica is common in patients with developmental disabilities and can be life-threatening. It is important to identify pica and manage it appropriately.

CASE REPORT: A 19-year-old male presented with history of abdominal pain and vomiting for two days. On imaging he was found to have multiple foreign bodies within the small and large bowel. After trial of conservative management, he was taken up for exploratory laparotomy. He was found to have trichobezoar and lithobezoar obstructing the bowel at terminal ileum. Bezoars when removed via enterotomy.

DISCUSSION: Bezoars are conglomerates of non-absorbable food or fibre formed in the alimentary tract. Trichobezoar forms following ingestion of hair and usually leads to gastric outlet obstruction. Lithobezoar refers to the accumulation of stones in the GIT. Reports of colonic lithobezoar are rare. Pica is the persistent ingestion of non-nutritive substances beyond a developmentally inappropriate age. Patients should be referred to a psychologist/behaviour analyst and caregivers should also be educated.

\section{INTRODUCTION}

Pica is defined as the persistent ingestion of non-nutritive substances for at least 1 month at $>2$ years of age. It is the most common eating disorder seen among developmentally challenged individuals and can also be considered the most dangerous form of self-injurious behaviour. Pica can lead to malnutrition and acute life-threatening complications like hollow viscus perforation and intestinal obstruction.

Gastro-intestinal tract obstruction can occur following the ingestion of foreign bodies and/or bezoar formation. Bezoars are conglomerates of food or fibre that form in the alimentary tract. Commonly found are phytobezoars (elderly and postgastrectomy patients), lactobezoars (seen in neonates) and trichobezoars (seen in adolescent females with trichotil lomania). Obstruction usually occurs at level of gastric outlet in cases of trichobezoars - these are usually adolescent females having trichotillomania. Lithobezoars which formed by accumulation of ingested stones, have been rarely reported in literature.

Here we present a case of a young adult male who presented with abdominal pain and was found to have untreated pica leading to trichobezoar and lithobezoar formation that had caused small bowel obstruction.

\section{CASE REPORT:}

The patient was a 19-year-old male, who presented with complaints of abdominal pain and vomiting for two days. The pain was localized to lower abdomen, would increase following food intake and had progressively increased in intensity. He had had 5-8 episodes of vomiting (non-bilious vomitus) per day. The patient had also not passed stools for 2 days; however, he was passing flatus. There was no history of any previously diagnosed medical illness or surgery. Parents gave history suggestive of undiagnosed mental illness since childhood - the patient had delayed attainment of developmental milestones, poor scholastic performance and occasionally displayed aggressive behaviour. He would also occasionally consume alcohol and tobacco when not supervised. The parents' marriage was consanguineous. No other significant family history.

On examination, the patient was conscious, oriented to time, place and person and cooperated well. He was afebrile and hemodynamically stable (PR: $88 \mathrm{bpm}, \mathrm{BP}: 100 / 80 \mathrm{mmHg}$ ). There was no pallor, icterus, cyanosis, clubbing, generalized lymphadenopathy or pedal oedema. Breakage and loss of multiple teeth were noted upon examination of the oral cavity. The abdomen was soft, with no distension; tenderness present over right iliac fossa and bowel sounds heard. On per rectal examination, the sphincter tone was normal, rectum empty and mucosa free. Other systemic examination revealed no abnormality.

Based on history and examination, a provisional diagnosis of acute appendicitis was made and we proceeded with investigations. Routine blood investigations revealed elevated total leukocyte count and no other abnormalities. Ultrasound of abdomen and pelvis revealed dilated small bowel loops showing to and fro peristalsis, prominent large bowel loops; multiple echogenic foci with posterior acoustic shadowing were noted within small and large bowel lumen features were suggestive of small bowel obstruction. X-ray abdomen erect was taken which showed multiple foreign bodies (?pebbles) within the small and large bowel.

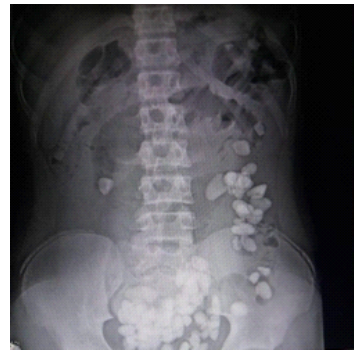

Figure 1 X-Ray Erect Abdomen taken on day of admission 
On further probing, parents gave a history of pica. There had been repeated incidents of the patient ingesting small stones when unsupervised and stones would be found in his stool.

Based on imaging findings a diagnosis of lithobezoar causing subacute small bowel obstruction was made. The patient was kept nil per oral with Ryle's tube in situ and observed for 48 hrs. The patient did not pass flatus or stools and repeat X-rays showed no improvement. Hence, he was taken up for exploratory laparotomy.

Intra-operatively, small bowel loops found to be dilated with a transition zone in terminal ileum where a soft "mass" was palpable within the bowel (?foreign body). Enterotomy was done (longitudinal incision) and a trichobezoar was found along with multiple smooth-edged pebbles proximally. Small bowel contents up to IC junction were milked out and enterotomy was closed in a transverse orientation.

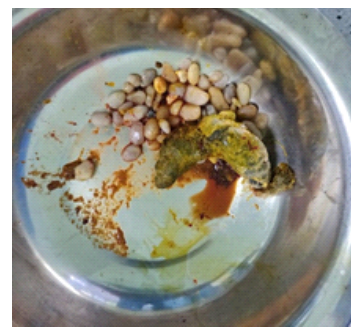

Figure 2 Pebbles and trichobezoar (stained with bowel contents) removed through jejunal enterotomy

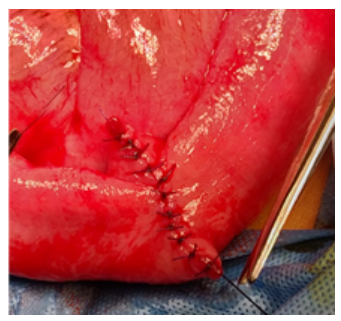

Figure 3 Enterotomy incision after closing in transverse manner

The patient tolerated the procedure well. Remaining stones within the large bowel passed once the patient resumed bowel movements by POD\#4.

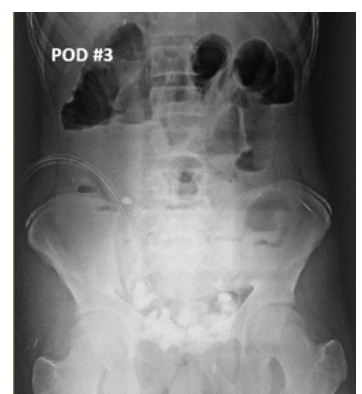

Figure $4 X$-Ray Erect abdomen taken on POD \#3

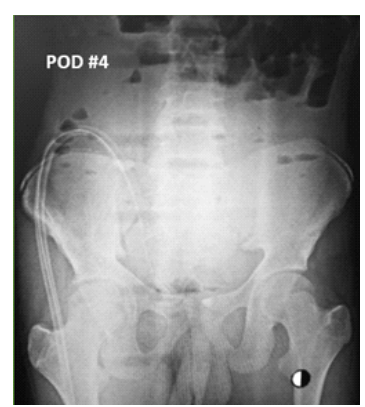

Figure $5 X$-Ray Erect Abdomen taken on POD \#4
He was then restarted on normal oral diet. There were no postoperative complications. Psychiatry opinion was taken and he was diagnosed with intellectual disability with behavioural problems. He was started on risperidone and advised regular follow up.

\section{DISCUSSION}

Bezoars are conglomerates of food or fibre that form in the alimentary tract. They increase in size by accretion of nonabsorbable food or fibres. Most commonly found are phytobezoars, usually among the elderly and in postgastrectomy patients. Other common types are lactobezoars (seen in neonates) and trichobezoars. Trichobezoar formation typically occurs due to trichotillomania-a disorder of compulsive plucking of hair seen most commonly in female children and adolescent females (prevalence: around $1 \%$ of the total population). One-third of these patients have trichophagia, and just $1 \%$ of them eat enough hair to accumulate trichobezoars requiring surgical intervention. Trichobezoars are most commonly found within the stomach. Only $15 \%$ of cases are found in the small bowel. The most common sites of obstruction are the gastric outlet or duodenum. Obstruction of the bowel is quite rare.

Lithobezoar refers to the accumulation of stones in the GIT. The most common complication presented with was intestinal obstruction, followed by perforation peritonitis. It is possible to elicit them clinically. On palpation, there may be a "colonic crunch sign" and "colonic crush sign". A radiograph will confirm the diagnosis; the appearance is characteristically described as "corn on the cob". Lithobezoars are rare - their exact prevalence is unknown; Only 11 case reports of colonic lithobezoar were found on literature search - all occurred in young children presenting with acute abdomen.

Most children learn to differentiate non-food substances by 2 years of age. Pica is defined as the persistent ingestion of nonnutritive substances for at least 1 month at an age when it is developmentally inappropriate. It is commonly associated with deficiency of iron (and other minerals). However, it is not clear whether pica is a symptom or cause of the said deficiency. Pica is also seen in people with developmental disabilities, mental illnesses, sickle cell anaemia, those undergoing chronic dialysis, and in pregnancy. Pica is the most common eating disorder seen among the develop mentally disabled. Prevalence estimates range from 5.7 to $25.8 \%$. It can be considered the most dangerous among selfinjurious behaviours exhibited by this population. Pica can manifest as poisoning, parasitic infestation, tooth loss/ abrasion, constipation, ulcerations, perforation, intestinal obstruction and can be lethal.

Most of these patients present with complications, not after their first episode of pica, but have a long history that has not been adequately handled or even identified. Hence it is important that once recognized, this condition is effectively managed. Ideally, once pica is identified, the patient should be referred to a psychologist/behaviour analyst. Behaviour modification techniques including Physical restraint; Negative practice; Overcorrection; Aversive stimulation may be used. Caregivers should be educated about pica and trained for its prevention. Patients' environment should be kept free of potentially dangerous objects. The aim should be the complete cessation of pica episodes to prove therapy as effective.

\section{CONCLUSION:}

A strong suspicion of pica should be maintained when evaluating developmentally disabled individuals. On diagnosis of pica, patient should undergo psychiatric evaluation and behaviour modification therapy as well as medication if needed to prevent recurrent episodes. Strict supervision at place of residence is also advisable. 


\section{REFERENCES:}

1. American Psychiatric Association. DSM-IV-TR: Diagnostic and Statistical Manual of Mental Disorders, Text Revision. American Psychiatric Press. 2000:103-105.

2. Williams DE, McAdam D. Assessment, behavioral treatment, and prevention of pica: clinical guidelines and recommendations for practitioners. Res Dev Disabil. 2012 Nov-Dec. 33(6):2050-7.

3. Malhotra A, Jones L, Drugas G. Simultaneous gastric and small intestinal trichobezoars. Pediatr Emerg Care.2008 Nov.24(11):774-6.

4. Iwamuro M, et al. (2015). Review of the diagnosis and management of gastrointestinal bezoars.

5. Ibrahim OR, Lugga AS, Ibrahim N, Ibrahim LM, Suleiman BM. Iron-deficiency anemia with lithobezoar (pica): a rare cause of intestinal obstruction in a 5year-old Nigerian child. Int Med Case Rep J.2018;11:225-228.

6. Tokue H, Takahashi Y, Hirasawa S, et al. Intestinal obstruction in a mentally retarded patient due to pica. Ann Gen Psychiatry. 2015; 14:22. Published 2015 Jul 31.doi:10.1186/s12991-015-0060-4

7. Narayanan, S. K. Akbar Sherif, V. S., Babu, P. R., \& Nandakumar, T. K. (2008) Intestinal obstruction secondary to a colonic lithobezoar. Journal of Pediatric Surgery, 43(7), e9-e10.doi:10.1016/j.jpedsurg.2008.02.065

8. Numanoglu, K. V., \& Tatli, D. (2008). A rare cause of partial intestinal obstruction in a child: colonic lithobezoar. Emergency Medicine Journal, 25(5),312-313.doi:10.1136/emj.2007.056812 